\title{
Nonoscillatory Solutions to Higher-Order Nonlinear Neutral Dynamic Equations
}

\author{
Yang-Cong Qiu ${ }^{1} \mathbb{C}$, Irena Jadlovská ${ }^{2}$, Dhaou Lassoued ${ }^{3}$ and Tongxing $\mathrm{Li}^{4,5, *}$ \\ 1 School of Humanities, Shunde Polytechnic, Foshan 528333, China; q840410@163.com \\ 2 Department of Mathematics and Theoretical Informatics, Faculty of Electrical Engineering and Informatics, \\ Technical University of Košice, B. Němcovej 32, 04200 Košice, Slovakia; irena.jadlovska@tuke.sk \\ 3 Département de Mathématiques, Faculté des Sciences de Gabès, Université de Gabès, Cité Erriadh, \\ Gabès 6072, Tunisia; dhaou06@gmail.com \\ 4 LinDa Institute of Shandong Provincial Key Laboratory of Network Based Intelligent Computing, \\ Linyi University, Linyi 276005, China \\ 5 School of Information Science and Engineering, Linyi University, Linyi 276005, China \\ * Correspondence: litongx2007@163.com
}

Received: 31 December 2018; Accepted: 25 February 2019; Published: 28 February 2019

\begin{abstract}
For a class of nonlinear higher-order neutral dynamic equations on a time scale, we analyze the existence and asymptotic behavior of nonoscillatory solutions on the basis of hypotheses that allow applications to equations with different integral convergence and divergence of the reciprocal of the coefficients. Two examples are presented to demonstrate the efficiency of new results.
\end{abstract}

Keywords: nonoscillatory behavior; existence; nonlinear neutral dynamic equation; higher-order; time scale

\section{Introduction}

In this article, we investigate the existence and asymptotic behavior of nonoscillatory solutions to a class of dynamic equations on a time scale $\mathbb{T}$

$$
R_{n}(t, x(t))+f(t, x(h(t)))=0,
$$

where $\sup \mathbb{T}=\infty, t \in\left[t_{0}, \infty\right)_{\mathbb{T}}$ with $t_{0} \in \mathbb{T}, n \geq 3$, and

$$
R_{k}(t, x(t))=\left\{\begin{array}{lc}
x(t)+p(t) x(g(t)), & k=0, \\
r_{n-k}(t) R_{k-1}^{\Delta}(t, x(t)), & 1 \leq k \leq n-1, \\
R_{n-1}^{\Delta}(t, x(t)), & k=n .
\end{array}\right.
$$

Definition 1. As is customary in this field, a solution of Equation (1) is termed nonoscillatory provided that $x$ is either eventually positive or eventually negative; otherwise, it is said to be oscillatory.

We refer the reader to [1-6], where the fundamental theory of time scales was investigated. In the last few years, the analysis of oscillatory and nonoscillatory behavior of differential and difference equations has been unified, extended, and generalized by corresponding theory of dynamic equations on time scales; see, for instance, Refs. [7-24]. Some conclusions for the existence and asymptotic behavior of nonoscillatory solutions to various classes of neutral dynamic equations have been shown in [11-13,16,19-22,24]. Zhu and Wang [24] studied a dynamic equation

$$
[x(t)+p(t) x(g(t))]^{\Delta}+f(t, x(h(t)))=0
$$


and established several criteria for the existence of the solutions via the Krasnoselskii's fixed point theorem. As a matter of fact, Equation (2) can be regarded as Equation (1) in the case when $n=1$. In the special case when $n=2$, Equation (1) reduces to a dynamic equation

$$
\left[r(t)(x(t)+p(t) x(g(t)))^{\Delta}\right]^{\Delta}+f(t, x(h(t)))=0,
$$

which was examined by Deng and Wang [11] and Gao and Wang [13]. The different assumptions $\int_{t_{0}}^{\infty} 1 / r(t) \Delta t=\infty$ in [11] and $\int_{t_{0}}^{\infty} 1 / r(t) \Delta t<\infty$ in [13] cause a phenomenon that the asymptotic behavior of nonoscillatory solutions to Equation (3) is greatly different. Moreover, it is clear that the asymptotic behavior is more complicated assuming that $\int_{t_{0}}^{\infty} 1 / r(t) \Delta t=\infty$.

To find a more general rule of the existence and asymptotic behavior of nonoscillatory solutions to Equation (1), Qiu [19] considered Equation (1) in the special case where $n=3$, namely,

$$
\left(r_{1}(t)\left(r_{2}(t)(x(t)+p(t) x(g(t)))^{\Delta}\right)^{\Delta}\right)^{\Delta}+f(t, x(h(t)))=0
$$

with $\int_{t_{0}}^{\infty} 1 / r_{1}(t) \Delta t=\int_{t_{0}}^{\infty} 1 / r_{2}(t) \Delta t=\infty$. The author introduced two functions

$$
R_{1}(t)=1+\int_{t_{0}}^{t} \frac{1}{r_{2}(s)} \Delta s \quad \text { and } \quad R_{2}(t)=1+\int_{t_{0}}^{t} \int_{t_{0}}^{s} \frac{1}{r_{1}(u) r_{2}(s)} \Delta u \Delta s
$$

to divide the eventually positive solutions of Equation (4) into five groups, and presented some existence conditions of them, respectively.

Qiu and Wang [20] were concerned with Equation (1) under the conditions $\int_{t_{0}}^{\infty} 1 / r_{i}(t) \Delta t<\infty$ for $i=1,2, \ldots, n-1$, which include Equation (4) when $n=3$ with $\int_{t_{0}}^{\infty} 1 / r_{i}(t) \Delta t<\infty$ for $i=1,2$. It shows that there exist only two cases that $\lim _{t \rightarrow \infty} x(t)=b>0$ and $\lim _{t \rightarrow \infty} x(t)=0$, where $x$ is assumed to be an eventually positive solution of Equation (1). Furthermore, this result can be extended to [13] when $n=2$ and [24] when $n=1$.

When the convergence and divergence of the integrals $\int_{t_{0}}^{\infty} 1 / r_{i}(t) \Delta t$ for $i=1,2, \ldots, n-1$ are different, for Equation (4), there exist two cases as follows:

(B1) $\int_{t_{0}}^{\infty} 1 / r_{1}(t) \Delta t=\infty$ and $\int_{t_{0}}^{\infty} 1 / r_{2}(t) \Delta t<\infty$;

(B2) $\int_{t_{0}}^{\infty} 1 / r_{1}(t) \Delta t<\infty$ and $\int_{t_{0}}^{\infty} 1 / r_{2}(t) \Delta t=\infty$.

Qiu et al. considered the case (B1) in [22] and the case (B2) in [21], successively. The conclusions complement the results in $[19,20]$ when $n=3$.

For Equation (1), it is significant to continue to investigate more general cases of the convergence and divergence of the integrals $\int_{t_{0}}^{\infty} 1 / r_{i}(t) \Delta t$ for $i=1,2, \ldots, n-1$. Throughout, we assume that the following hypotheses are satisfied:

$(C 1) r_{i} \in \mathrm{C}_{\mathrm{rd}}\left(\left[t_{0}, \infty\right)_{\mathbb{T}},(0, \infty)\right), i=1,2, \ldots, n-1$, and there are constants $M_{i}>0, i=2,3, \ldots, n-1$ such that

$$
\int_{t_{0}}^{\infty} \frac{\Delta t}{r_{1}(t)}=\infty \quad \text { and } \quad \int_{t_{0}}^{\infty} \frac{\Delta t}{r_{i}(t)}=M_{i}<\infty, \quad i=2,3, \ldots, n-1 ;
$$

$(C 2) p \in \mathrm{C}_{\mathrm{rd}}\left(\left[t_{0}, \infty\right)_{\mathbb{T}}, \mathbb{R}\right)$ and $\lim _{t \rightarrow \infty} p(t)=p_{0}$, where $\left|p_{0}\right|<1$;

$(C 3) g, h \in C_{\text {rd }}\left(\left[t_{0}, \infty\right)_{\mathbb{T}}, \mathbb{T}\right), g(t) \leq t, \lim _{t \rightarrow \infty} g(t)=\lim _{t \rightarrow \infty} h(t)=\infty$, and if $p_{0} \in(-1,0]$, then there exists a sequence $\left\{c_{k}\right\}_{k \geq 0}$ satisfying $\lim _{k \rightarrow \infty} c_{k}=\infty$ and $g\left(c_{k+1}\right)=c_{k}$;

(C4) $f \in \mathrm{C}\left(\left[t_{0}, \infty\right)_{\mathbb{T}} \times \mathbb{R}, \mathbb{R}\right)$ is nondecreasing in $x$ and $x f(t, x)>0$ for $x \neq 0$;

(C5) if

$$
\int_{t_{0}}^{\infty} \int_{t_{0}}^{u_{n-1}} \int_{t_{0}}^{u_{n-2}} \cdots \int_{t_{0}}^{u_{2}} \frac{1}{\prod_{i=1}^{n-1} r_{i}\left(u_{i}\right)} \Delta u_{1} \Delta u_{2} \cdots \Delta u_{n-1}=\infty,
$$


then define

$$
R(t)=1+\int_{t_{0}}^{t} \int_{t_{0}}^{u_{n-1}} \int_{t_{0}}^{u_{n-2}} \cdots \int_{t_{0}}^{u_{2}} \frac{1}{\prod_{i=1}^{n-1} r_{i}\left(u_{i}\right)} \Delta u_{1} \Delta u_{2} \cdots \Delta u_{n-1}
$$

where

$$
\lim _{t \rightarrow \infty} \frac{R(g(t))}{R(t)}=\eta \in(0,1]
$$

is supposed to hold.

In view of the results established in [11,13], it is not difficult to see that the existence and asymptotic behavior of nonoscillatory solutions to Equation (1) are more complex than those in [20]. Therefore, the criteria obtained in this article develop and improve some known conclusions reported in the references. Finally, we present two examples to demonstrate the versatility of new results.

\section{Auxiliary Results}

To establish criteria for the existence of nonoscillatory solutions to Equation (1), we need a Banach space and Krasnoselskii's fixed point theorem as follows.

Definition 2. Letting $\lambda=0,1$, define a Banach space

$$
\mathrm{BC}_{\lambda}\left[T_{0}, \infty\right)_{\mathbb{T}}=\left\{x: x \in \mathrm{C}\left(\left[T_{0}, \infty\right)_{\mathbb{T}}, \mathbb{R}\right) \text { and } \sup _{t \in\left[T_{0}, \infty\right)_{\mathbb{T}}}\left|\frac{x(t)}{R^{2 \lambda}(t)}\right|<\infty\right\}
$$

with the norm

$$
\|x\|_{\lambda}=\sup _{t \in\left[T_{0}, \infty\right)_{\mathbb{T}}}\left|\frac{x(t)}{R^{2 \lambda}(t)}\right|
$$

where $\mathrm{C}\left(\left[T_{0}, \infty\right)_{\mathbb{T}}, \mathbb{R}\right)$ is the set containing all continuous functions mapping $\left[T_{0}, \infty\right)_{\mathbb{T}}$ into $\mathbb{R}$.

Lemma 1. (Krasnoselskii's fixed point theorem) Let $\Omega$ be a bounded, convex, and closed subset of a Banach space $X$. Assume that there are two operators $U, S: \Omega \rightarrow X$ such that $U$ is contractive, $S$ is completely continuous, and $U x+S y \in \Omega$ for all $x, y \in \Omega$. Then, $U+S$ has a fixed point in $\Omega$.

Define $z(t)=x(t)+p(t) x(g(t))$. Without loss of generality, we consider only the eventually positive solutions of Equation (1). Then, we have the following lemma (see [12] (Lemma 2.3) and [22] (Lemma 2.1)).

Lemma 2. Let $x$ be an eventually positive solution of Equation (1) and

$$
\lim _{t \rightarrow \infty} \frac{z(t)}{R^{\lambda}(t)}=a, \quad \lambda=0,1,
$$

where $\lambda=1$ only if condition (5) holds. Suppose that a is finite. Then,

$$
\lim _{t \rightarrow \infty} \frac{x(t)}{R^{\lambda}(t)}=\frac{a}{1+p_{0} \eta^{\lambda}}
$$

or $x / R^{\lambda}$ is unbounded.

For the sake of simplicity, we give a classification to divide all eventually positive solutions of Equation (1) into four types.

Theorem 1. Let $x$ be an eventually positive solution of Equation (1). Then, there are four possible types for $x$ : 
(A1) $\lim _{t \rightarrow \infty} x(t)=0$;

(A2) $\lim _{t \rightarrow \infty} x(t)=b$ for some constant $b>0$;

(A3) $\lim _{t \rightarrow \infty} x(t)=\infty$ and $\lim _{t \rightarrow \infty} x(t) / R(t)=b$, where $b>0$ is a constant;

(A4) $\lim \sup _{t \rightarrow \infty} x(t)=\infty$ and $\lim _{t \rightarrow \infty} x(t) / R(t)=0$.

Proof. Let $x$ be an eventually positive solution of Equation (1). By virtue of (C2) and (C3), there exist a $t_{1} \in\left[t_{0}, \infty\right)_{\mathbb{T}}$ and $\left|p_{0}\right|<p_{1}<1$ satisfying $x(t)>0, x(g(t))>0, x(h(t))>0$, and $|p(t)| \leq p_{1}$ for $t \in\left[t_{1}, \infty\right)_{\mathbb{T}}$. For $t \in\left[t_{1}, \infty\right)_{\mathbb{T}}$, we get

$$
R_{n-1}^{\Delta}(t, x(t))=-f(t, x(h(t)))<0,
$$

which implies that $R_{n-1}(t, x(t))=r_{1}(t) R_{n-2}^{\Delta}(t, x(t))$ is strictly decreasing on $\left[t_{1}, \infty\right)_{\mathbb{T}}$. Then, we need to consider two cases.

Case 1. Suppose first that $R_{n-2}^{\Delta}$ is eventually negative. Then,

$$
\lim _{t \rightarrow \infty} r_{1}(t) R_{n-2}^{\Delta}(t, x(t))=L_{2}
$$

where $-\infty \leq L_{2}<0$. Hence, there exist a constant $c_{1}<0$ and a $t_{2} \in\left[t_{1}, \infty\right)_{\mathbb{T}}$ such that $r_{1}(t) R_{n-2}^{\Delta}(t, x(t)) \leq c_{1}$ for $t \in\left[t_{2}, \infty\right)_{\mathbb{T}}$, which yields

$$
R_{n-2}^{\Delta}(t, x(t)) \leq \frac{c_{1}}{r_{1}(t)}, \quad t \in\left[t_{2}, \infty\right)_{\mathbb{T}} .
$$

Integrating inequality (7) from $t_{2}$ to $t, t \in\left[\sigma\left(t_{2}\right), \infty\right)_{\mathbb{T}}$, we deduce that

$$
R_{n-2}(t, x(t))-R_{n-2}\left(t_{2}, x\left(t_{2}\right)\right) \leq c_{1} \int_{t_{2}}^{t} \frac{\Delta s}{r_{1}(s)} .
$$

In view of $(C 1)$, letting $t \rightarrow \infty$, we obtain $R_{n-2}(t, x(t))=r_{2}(t) R_{n-3}^{\Delta}(t, x(t)) \rightarrow-\infty$, which means that $R_{n-3}^{\Delta}$ is negative and $R_{n-3}$ is strictly decreasing for large $t$. When $n=3, z$ is nonoscillatory. We can declare that

$$
\lim _{t \rightarrow \infty} z(t)=L_{0}
$$

where $0 \leq L_{0}<\infty$. Do not assume it; that is, $\lim _{t \rightarrow \infty} z(t)<0$. Then, we have $p_{0} \in(-1,0]$ and so there exists a $t_{3} \in\left[t_{2}, \infty\right)_{\mathbb{T}}$ such that

$$
x(t)<-p(t) x(g(t)) \leq p_{1} x(g(t)), \quad t \in\left[t_{3}, \infty\right)_{\mathbb{T}} .
$$

It follows from (C3) that there is a positive integer $k_{0}$ such that $c_{k} \in\left[t_{3}, \infty\right)_{\mathbb{T}}$ for all $k \geq k_{0}$. For any given $k \geq k_{0}+1$, we always arrive at

$$
x\left(c_{k}\right)<p_{1} x\left(g\left(c_{k}\right)\right)=p_{1} x\left(c_{k-1}\right)<p_{1}^{2} x\left(c_{k-2}\right)<\cdots<p_{1}^{k-k_{0}} x\left(c_{k_{0}}\right),
$$

which yields $\lim _{k \rightarrow \infty} x\left(c_{k}\right)=0$ and $\lim _{k \rightarrow \infty} z\left(c_{k}\right)=0$. This contradicts the assumption, and so equality (8) holds.

When $n \geq 4$, since $R_{n-3}(t, x(t))=r_{3}(t) R_{n-4}^{\Delta}(t, x(t))$ is eventually strictly decreasing, there exists a $t_{4} \in\left[t_{2}, \infty\right)_{\mathbb{T}}$ such that for $t \in\left[t_{4}, \infty\right)_{\mathbb{T}}$, we have

$$
R_{n-4}^{\Delta}(t, x(t)) \leq \frac{r_{3}\left(t_{4}\right) R_{n-4}^{\Delta}\left(t_{4}, x\left(t_{4}\right)\right)}{r_{3}(t)}
$$

If there is a $t_{5} \in\left[t_{4}, \infty\right)_{\mathbb{T}}$ such that $R_{n-4}^{\Delta}\left(t_{5}, x\left(t_{5}\right)\right) \leq 0$, then $r_{3}(t) R_{n-4}^{\Delta}(t, x(t)) \leq 0$ for $t \in\left[t_{5}, \infty\right)_{\mathbb{T}}$ and thus $R_{n-4}^{\Delta}$ is eventually negative. Otherwise, if $R_{n-4}^{\Delta}(t, x(t))>0$ for all $t \in\left[t_{4}, \infty\right)_{\mathbb{T}}$, then $R_{n-4}^{\Delta}$ is 
eventually positive. Hence, $R_{n-4}$ is always eventually monotonic. Integrating inequality (9) from $t_{4}$ to $t, t \in\left[\sigma\left(t_{4}\right), \infty\right)_{\mathbb{T}}$, we conclude that

$$
R_{n-4}(t, x(t))-R_{n-4}\left(t_{4}, x\left(t_{4}\right)\right) \leq r_{3}\left(t_{4}\right) R_{n-4}^{\Delta}\left(t_{4}, x\left(t_{4}\right)\right) \int_{t_{4}}^{t} \frac{\Delta s}{r_{3}(s)} \leq r_{3}\left(t_{4}\right)\left|R_{n-4}^{\Delta}\left(t_{4}, x\left(t_{4}\right)\right)\right| \cdot M_{3},
$$

which implies that $R_{n-4}$ is upper bounded. If $n=4$, then we see that $z$ is eventually monotonic and upper bounded, and equality (8) holds.

When $n \geq 5$, since $R_{n-4}(t, x(t))$ (or $r_{4}(t) R_{n-5}^{\Delta}(t, x(t))$ ) is eventually monotonic, $R_{n-5}^{\Delta}$ is nonoscillatory. It follows that $R_{n-5}$ is eventually monotonic. Noticing that $R_{n-4}$ is upper bounded, there exist a constant $c_{2}$ and a $t_{6} \in\left[t_{4}, \infty\right)_{\mathbb{T}}$ such that for $t \in\left[t_{6}, \infty\right)_{\mathbb{T}}$,

$$
R_{n-4}(t, x(t))=r_{4}(t) R_{n-5}^{\Delta}(t, x(t)) \leq c_{2},
$$

which yields

$$
R_{n-5}^{\Delta}(t, x(t)) \leq \frac{c_{2}}{r_{4}(t)}, \quad t \in\left[t_{6}, \infty\right)_{\mathbb{T}}
$$

Integrating inequality (10) from $t_{6}$ to $t, t \in\left[\sigma\left(t_{6}\right), \infty\right)_{\mathbb{T}}$, we get

$$
R_{n-5}(t, x(t))-R_{n-5}\left(t_{6}, x\left(t_{6}\right)\right) \leq c_{2} \int_{t_{6}}^{t} \frac{\Delta s}{r_{4}(s)} \leq\left|c_{2}\right| \cdot M_{4}
$$

which means that $R_{n-5}$ is upper bounded. If $n=5$, then we deduce that equality (8) holds similarly. Analogously, for $n \geq 3$, it follows that equality (8) always holds. Therefore, by virtue of Lemma 2, we conclude that $(A 1)$ or $(A 2)$ holds.

Case 2. Assume now that $R_{n-2}^{\Delta}$ is eventually positive. Then,

$$
\lim _{t \rightarrow \infty} r_{1}(t) R_{n-2}^{\Delta}(t, x(t))=L_{2}
$$

where $0 \leq L_{2}<\infty$. We consider the following two cases:

$$
\lim _{t \rightarrow \infty} r_{1}(t) R_{n-2}^{\Delta}(t, x(t))=b>0 \quad \text { and } \quad \lim _{t \rightarrow \infty} r_{1}(t) R_{n-2}^{\Delta}(t, x(t))=0 .
$$

If $\lim _{t \rightarrow \infty} r_{1}(t) R_{n-2}^{\Delta}(t, x(t))=b>0$, then there is a $t_{2} \in\left[t_{1}, \infty\right)_{\mathbb{T}}$ such that for $t \in\left[t_{2}, \infty\right)_{\mathbb{T}}$,

$$
\left(r_{2}(t) R_{n-3}^{\Delta}(t, x(t))\right)^{\Delta}>\frac{b}{r_{1}(t)} .
$$

Integrating inequality (11) from $t_{2}$ to $t, t \in\left[\sigma\left(t_{2}\right), \infty\right)_{\mathbb{T}}$, we arrive at

$$
r_{2}(t) R_{n-3}^{\Delta}(t, x(t))>r_{2}\left(t_{2}\right) R_{n-3}^{\Delta}\left(t_{2}, x\left(t_{2}\right)\right)+b \int_{t_{2}}^{t} \frac{\Delta s}{r_{1}(s)} .
$$

By virtue of $(\mathrm{C} 1), r_{2}(t) R_{n-3}^{\Delta}(t, x(t)) \rightarrow \infty$ as $t \rightarrow \infty$, which implies that $R_{n-3}^{\Delta}$ is positive and $R_{n-3}$ is strictly increasing for large $t$. Thus, $R_{n-3}$ is nonoscillatory. When $n=3, R_{n-3}=z$. As before, we have

$$
\lim _{t \rightarrow \infty} z(t)=L_{0}
$$

where $0 \leq L_{0} \leq \infty$. When $n \geq 4$, since $R_{n-3}(t, x(t))=r_{3}(t) R_{n-4}^{\Delta}(t, x(t))$, we deduce that $R_{n-4}^{\Delta}(t, x(t))$ is nonoscillatory, and $R_{n-4}$ is eventually monotonic. If $n=4$, then $R_{n-4}=z$, and equality (12) holds. When $n \geq 5$, it follows that $R_{n-5}$ is eventually monotonic similarly. Analogously, for $n \geq 3$, it follows that equality (12) always holds. 
If $\lim _{t \rightarrow \infty} r_{1}(t) R_{n-2}^{\Delta}(t, x(t))=0$, since $R_{n-2}^{\Delta}=\left(r_{2} R_{n-3}^{\Delta}\right)^{\Delta}$ is eventually positive, then

$$
\lim _{t \rightarrow \infty} r_{2}(t) R_{n-3}^{\Delta}(t, x(t))=L_{1}
$$

where $-\infty<L_{1} \leq \infty$. Moreover, $r_{2} R_{n-3}^{\Delta}$ is strictly increasing for large $t$. It follows that $R_{n-3}^{\Delta}$ is nonoscillatory. Thus, $R_{n-3}$ is always eventually monotonic and nonoscillatory. Similarly as before, we deduce that $\lim _{t \rightarrow \infty} z(t)=L_{0} \geq 0$ when $n \geq 3$.

When $L_{1}=\infty$, we get $0 \leq L_{0} \leq \infty$ similarly as before. If $-\infty<L_{1}<\infty$, then there exist a constant $d_{1}>0$ and a $t_{3} \in\left[t_{1}, \infty\right)_{\mathbb{T}}$ such that $r_{2}(t) R_{n-3}^{\Delta}(t, x(t)) \leq d_{1}$ for $t \in\left[t_{3}, \infty\right)_{\mathbb{T}}$, which yields

$$
R_{n-3}^{\Delta}(t, x(t)) \leq \frac{d_{1}}{r_{2}(t)}, \quad t \in\left[t_{3}, \infty\right)_{\mathbb{T}}
$$

Integrating inequality (13) from $t_{3}$ to $t, t \in\left[\sigma\left(t_{3}\right), \infty\right)_{\mathbb{T}}$, we have

$$
R_{n-3}(t, x(t))-R_{n-3}\left(t_{3}, x\left(t_{3}\right)\right) \leq d_{1} \int_{t_{3}}^{t} \frac{\Delta s}{r_{2}(s)} \leq d_{1} \cdot M_{2}
$$

When $n=3, R_{n-3}=z$, and so $z$ is upper bounded. When $n \geq 4$, there exist a constant $d_{2}>0$ and a $t_{4} \in\left[t_{3}, \infty\right)_{\mathbb{T}}$ such that $r_{3}(t) R_{n-4}^{\Delta}(t, x(t)) \leq d_{2}$ for $t \in\left[t_{4}, \infty\right)_{\mathbb{T}}$. It follows that

$$
R_{n-4}^{\Delta}(t, x(t)) \leq \frac{d_{2}}{r_{3}(t)}, \quad t \in\left[t_{4}, \infty\right)_{\mathbb{T}} .
$$

Similarly, we see that $R_{n-4}$ is upper bounded. If $n=4$, then $R_{n-4}=z$, and thus $z$ is upper bounded. Analogously, for $n \geq 3$, we deduce that $z$ is always upper bounded. Hence, $0 \leq L_{0}<\infty$.

According to Lemma 2, if $0 \leq L_{0}<\infty$, then case $(A 1)$ or case $(A 2)$ holds; if $L_{0}=\infty$, then we obtain that $x$ is infinite. Furthermore, by virtue of L'Hôpital's rule (see [5] (Theorem 1.120)), we deduce that

$$
\lim _{t \rightarrow \infty} R_{n}(t, x(t))=\lim _{t \rightarrow \infty} \frac{z(t)}{R(t)}=L_{2}
$$

where $0 \leq L_{2}<\infty$. It follows that one of cases $(A 3)$ and $(A 4)$ holds.

The proof is complete.

\section{Main Results}

We establish several criteria for the existence of various types of eventually positive solutions of Equation (1). Firstly, suppose that

$$
\int_{t_{0}}^{\infty} \int_{t_{0}}^{u_{n-1}} \int_{t_{0}}^{u_{n-2}} \cdots \int_{t_{0}}^{u_{2}} \frac{1}{\prod_{i=1}^{n-1} r_{i}\left(u_{i}\right)} \Delta u_{1} \Delta u_{2} \cdots \Delta u_{n-1}<\infty,
$$

which means that condition (5) is not satisfied.

Theorem 2. Let condition (14) be fulfilled. Then, Equation (1) has an eventually positive solution $x$ satisfying $\lim _{t \rightarrow \infty} x(t)=b$ iff

$$
\int_{t_{0}}^{\infty} \int_{t_{0}}^{u_{n-1}} \int_{t_{0}}^{u_{n-2}} \cdots \int_{t_{0}}^{u_{1}} \frac{f\left(u_{0}, K\right)}{\prod_{i=1}^{n-1} r_{i}\left(u_{i}\right)} \Delta u_{0} \Delta u_{1} \cdots \Delta u_{n-1}<\infty
$$

for some constant $K>0$, where $b>0$ is a constant. 
Proof. Let $x$ be an eventually positive solution of Equation (1) that satisfies $\lim _{t \rightarrow \infty} x(t)=b>0$. Then, $\lim _{t \rightarrow \infty} z(t)=\left(1+p_{0}\right) b$, and there is a $t_{1} \in\left[t_{0}, \infty\right)_{\mathbb{T}}$ such that $x(t)>0, x(g(t))>0$, and $x(h(t)) \geq b / 2$ for $t \in\left[t_{1}, \infty\right)_{\mathbb{T}}$. Integrating Equation (1) from $t_{1}$ to $t, t \in\left[\sigma\left(t_{1}\right), \infty\right)_{\mathbb{T}}$, we arrive at

$$
R_{n-2}^{\Delta}(t, x(t))=\frac{R_{n-1}\left(t_{1}, x\left(t_{1}\right)\right)}{r_{1}(t)}-\frac{\int_{t_{1}}^{t} f\left(u_{0}, x\left(h\left(u_{0}\right)\right)\right) \Delta u_{0}}{r_{1}(t)} .
$$

Integrating equality (16) from $t_{1}$ to $t, t \in\left[\sigma\left(t_{1}\right), \infty\right)_{\mathbb{T}}$, we get

$$
\begin{aligned}
R_{n-2}(t, x(t))-R_{n-2}\left(t_{1}, x\left(t_{1}\right)\right) & =\int_{t_{1}}^{t} R_{n-2}^{\Delta}\left(u_{1}, x\left(u_{1}\right)\right) \Delta u_{1} \\
& =R_{n-1}\left(t_{1}, x\left(t_{1}\right)\right) \int_{t_{1}}^{t} \frac{\Delta u_{1}}{r_{1}\left(u_{1}\right)}-\int_{t_{1}}^{t} \int_{t_{1}}^{u_{1}} \frac{f\left(u_{0}, x\left(h\left(u_{0}\right)\right)\right)}{r_{1}\left(u_{1}\right)} \Delta u_{0} \Delta u_{1} .
\end{aligned}
$$

Similarly, for $n \geq 3$, we conclude that

$$
\begin{aligned}
z(t)-z\left(t_{1}\right)= & R_{1}\left(t_{1}, x\left(t_{1}\right)\right) \int_{t_{1}}^{t} \frac{1}{r_{n-1}\left(u_{n-1}\right)} \Delta u_{n-1} \\
& +\sum_{k=2}^{n-1} R_{k}\left(t_{1}, x\left(t_{1}\right)\right) \int_{t_{1}}^{t} \int_{t_{1}}^{u_{n-1}} \int_{t_{1}}^{u_{n-2}} \cdots \int_{t_{1}}^{u_{n-k+1}} \frac{1}{\prod_{i=n-k}^{n-1} r_{i}\left(u_{i}\right)} \Delta u_{n-k} \Delta u_{n-k+1} \cdots \Delta u_{n-1} \\
& -\int_{t_{1}}^{t} \int_{t_{1}}^{u_{n-1}} \int_{t_{1}}^{u_{n-2}} \cdots \int_{t_{1}}^{u_{1}} \frac{f\left(u_{0}, x\left(h\left(u_{0}\right)\right)\right)}{\prod_{i=1}^{n-1} r_{i}\left(u_{i}\right)} \Delta u_{0} \Delta u_{1} \cdots \Delta u_{n-1} .
\end{aligned}
$$

Letting $t \rightarrow \infty$, condition (14) holds if $n \geq 3$, and, when $n \geq 4$, for all $2 \leq k \leq n-2$, by virtue of (C1), we deduce that

$$
\int_{t_{1}}^{\infty} \int_{t_{1}}^{u_{n-1}} \int_{t_{1}}^{u_{n-2}} \cdots \int_{t_{1}}^{u_{n-k+1}} \frac{1}{\prod_{i=n-k}^{n-1} r_{i}\left(u_{i}\right)} \Delta u_{n-k} \Delta u_{n-k+1} \cdots \Delta u_{n-1} \leq \prod_{i=n-k}^{n-1} M_{i}<\infty .
$$

Hence,

$$
\int_{t_{1}}^{\infty} \int_{t_{1}}^{u_{n-1}} \int_{t_{1}}^{u_{n-2}} \cdots \int_{t_{1}}^{u_{1}} \frac{f\left(u_{0}, x\left(h\left(u_{0}\right)\right)\right)}{\prod_{i=1}^{n-1} r_{i}\left(u_{i}\right)} \Delta u_{0} \Delta u_{1} \cdots \Delta u_{n-1}<\infty
$$

Since $x(h(t)) \geq b / 2$, from $(C 4)$, it is obvious that

$$
\int_{t_{1}}^{\infty} \int_{t_{1}}^{u_{n-1}} \int_{t_{1}}^{u_{n-2}} \cdots \int_{t_{1}}^{u_{1}} \frac{f\left(u_{0}, b / 2\right)}{\prod_{i=1}^{n-1} r_{i}\left(u_{i}\right)} \Delta u_{0} \Delta u_{1} \cdots \Delta u_{n-1}<\infty,
$$

which implies that condition (15) holds.

Suppose that there exists some constant $K>0$ satisfying condition (15). Then, we will analyze two cases: (i) $0 \leq p_{0}<1$ and (ii) $-1<p_{0}<0$, respectively.

Case (i). $0 \leq p_{0}<1$. Take a constant $p_{1}$ such that $p_{0}<p_{1}<\left(1+4 p_{0}\right) / 5<1$. When $p_{0}>0$, by virtue of $(C 2)$ and condition (15), there is a $T_{0} \in\left[t_{0}, \infty\right)_{\mathbb{T}}$ such that for $t \in\left[T_{0}, \infty\right)_{\mathbb{T}}$,

$$
\begin{gathered}
p(t)>0, \quad \frac{5 p_{1}-1}{4} \leq p(t) \leq p_{1}<1, \\
\int_{T_{0}}^{\infty} \int_{T_{0}}^{u_{n-1}} \int_{T_{0}}^{u_{n-2}} \cdots \int_{T_{0}}^{u_{1}} \frac{f\left(u_{0}, K\right)}{\prod_{i=1}^{n-1} r_{i}\left(u_{i}\right)} \Delta u_{0} \Delta u_{1} \cdots \Delta u_{n-1} \leq \frac{\left(1-p_{1}\right) K}{8} .
\end{gathered}
$$

When $p_{0}=0$, choose a constant $p_{1}$ such that $|p(t)| \leq p_{1} \leq 1 / 13$ for $t \in\left[T_{0}, \infty\right)_{\mathbb{T}}$. By virtue of $(C 3)$, there is a $T_{1} \in\left(T_{0}, \infty\right)_{\mathbb{T}}$ such that $g(t) \geq T_{0}$ and $h(t) \geq T_{0}$ for $t \in\left[T_{1}, \infty\right)_{\mathbb{T}}$.

Let

$$
\Omega_{1}=\left\{x \in \mathrm{BC}_{0}\left[T_{0}, \infty\right)_{\mathbb{T}}: \frac{K}{2} \leq x(t) \leq K\right\}
$$


Clearly, $\Omega_{1}$ is a bounded, convex, and closed subset of $\mathrm{BC}_{0}\left[T_{0}, \infty\right)_{\mathbb{T}}$. Define now two operators $U_{1}, S_{1}: \Omega_{1} \rightarrow \mathrm{BC}_{0}\left[T_{0}, \infty\right)_{\mathbb{T}}$ by

$$
\begin{gathered}
\left(U_{1} x\right)(t)= \begin{cases}3 K p_{1} / 4-p(t) x(g(t)), & t \in\left[T_{1}, \infty\right)_{\mathbb{T}}, \\
\left(U_{1} x\right)\left(T_{1}\right), & t \in\left[T_{0}, T_{1}\right)_{\mathbb{T}},\end{cases} \\
\left(S_{1} x\right)(t)= \begin{cases}3 K / 4 & t \in\left[T_{1}, \infty\right)_{\mathbb{T}}, \\
+\int_{t}^{\infty} \int_{T_{1}}^{u_{n-1}} \int_{T_{1}}^{u_{n-2}} \cdots \int_{T_{1}}^{u_{1}} \frac{f\left(u_{0}, x\left(h\left(u_{0}\right)\right)\right)}{\prod_{i=1}^{n-1} r_{i}\left(u_{i}\right)} \Delta u_{0} \Delta u_{1} \cdots \Delta u_{n-1}, & t \in\left[T_{0}, T_{1}\right)_{\mathbb{T}} .\end{cases}
\end{gathered}
$$

The fact that $U_{1}$ and $S_{1}$ satisfy the conditions in Lemma 1 can be proved (see the proofs of [19] (Theorem 3.1) and [20] (Theorem 3.1)), and so is omitted. By virtue of Lemma 1, there is an $x \in \Omega_{1}$ such that $\left(U_{1}+S_{1}\right) x=x$. For $t \in\left[T_{1}, \infty\right)_{\mathbb{T}}$,

$$
x(t)=\frac{3\left(1+p_{1}\right) K}{4}-p(t) x(g(t))+\int_{t}^{\infty} \int_{T_{1}}^{u_{n-1}} \int_{T_{1}}^{u_{n-2}} \cdots \int_{T_{1}}^{u_{1}} \frac{f\left(u_{0}, x\left(h\left(u_{0}\right)\right)\right)}{\prod_{i=1}^{n-1} r_{i}\left(u_{i}\right)} \Delta u_{0} \Delta u_{1} \cdots \Delta u_{n-1} .
$$

Since $f\left(u_{0}, x\left(h\left(u_{0}\right)\right)\right) \leq f\left(u_{0}, K\right)$ for $u_{0} \in\left[T_{1}, \infty\right)_{\mathbb{T}}$, and

$$
\lim _{t \rightarrow \infty} \int_{t}^{\infty} \int_{T_{1}}^{u_{n-1}} \int_{T_{1}}^{u_{n-2}} \cdots \int_{T_{1}}^{u_{1}} \frac{f\left(u_{0}, K\right)}{\prod_{i=1}^{n-1} r_{i}\left(u_{i}\right)} \Delta u_{0} \Delta u_{1} \cdots \Delta u_{n-1}=0,
$$

we conclude that

$$
\lim _{t \rightarrow \infty} z(t)=\frac{3\left(1+p_{1}\right) K}{4} \text { and } \lim _{t \rightarrow \infty} x(t)=\frac{3\left(1+p_{1}\right) K}{4\left(1+p_{0}\right)}>0 .
$$

Case (ii). $-1<p_{0}<0$. Choose a constant $p_{1}$ satisfying $-p_{0}<p_{1}<\left(1-4 p_{0}\right) / 5<1$. By $($ C2 $)$ and condition (15), there is a $T_{0} \in\left[t_{0}, \infty\right)_{\mathbb{T}}$ such that $\left(5 p_{1}-1\right) / 4 \leq-p(t) \leq p_{1}<1$ for $t \in\left[T_{0}, \infty\right)_{\mathbb{T}}$. There also exists a $T_{1} \in\left(T_{0}, \infty\right)_{\mathbb{T}}$ such that $g(t) \geq T_{0}$ and $h(t) \geq T_{0}$ for $t \in\left[T_{1}, \infty\right)_{\mathbb{T}}$. Let $\mathrm{BC}_{0}\left[T_{0}, \infty\right)_{\mathbb{T}}$ and its subset $\Omega_{1}$ be as in (18). Define $S_{1}$ by (19) and $U_{1}^{\prime}$ on $\Omega_{1}$ by

$$
\left(U_{1}^{\prime} x\right)(t)= \begin{cases}-3 K p_{1} / 4-p(t) x(g(t)), & t \in\left[T_{1}, \infty\right)_{\mathbb{T}} \\ \left(U_{1}^{\prime} x\right)\left(T_{1}\right), & t \in\left[T_{0}, T_{1}\right)_{\mathbb{T}} .\end{cases}
$$

Similarly, as in the proofs of [19] (Theorem 3.1) and [20] (Theorem 3.1), we can prove that $U_{1}^{\prime}$ and $S_{1}$ satisfy the assumptions in Lemma 1 . Hence, there is an $x \in \Omega_{1}$ such that $\left(U_{1}^{\prime}+S_{1}\right) x=x$. For $t \in\left[T_{1}, \infty\right)_{\mathbb{T}}$,

$$
x(t)=\frac{3\left(1-p_{1}\right) K}{4}-p(t) x(g(t))+\int_{t}^{\infty} \int_{T_{1}}^{u_{n-1}} \int_{T_{1}}^{u_{n-2}} \cdots \int_{T_{1}}^{u_{1}} \frac{f\left(u_{0}, x\left(h\left(u_{0}\right)\right)\right)}{\prod_{i=1}^{n-1} r_{i}\left(u_{i}\right)} \Delta u_{0} \Delta u_{1} \cdots \Delta u_{n-1} .
$$

Letting $t \rightarrow \infty$, we deduce that

$$
\lim _{t \rightarrow \infty} z(t)=\frac{3\left(1-p_{1}\right) K}{4} \text { and } \lim _{t \rightarrow \infty} x(t)=\frac{3\left(1-p_{1}\right) K}{4\left(1+p_{0}\right)}>0 .
$$

This completes the proof.

Remark 1. Actually, the assumption (14) in Theorem 2 is not needed in the sufficiency of its proof. Thus, we obtain a corollary as follows.

Corollary 1. Assume that condition (15) is fulfilled for some constant $K>0$. Then, Equation (1) has an eventually positive solution $x$ satisfying $\lim _{t \rightarrow \infty} x(t)=b$, where $b>0$ is a constant. 
Now, we let

$$
A(\alpha)=\left\{x \in S: \lim _{t \rightarrow \infty} x(t)=\infty, \lim _{t \rightarrow \infty} \frac{x(t)}{R(t)}=\alpha\right\},
$$

where $S$ stands for the set containing all eventually positive solutions of Equation (1). Then, a lemma is presented as follows.

Lemma 3. Let $x$ be an eventually positive solution of Equation (1) such that $\lim _{t \rightarrow \infty} x(t)=\infty$. Then, condition (5) is satisfied, and $x \in A(0)$ or $x \in A(b)$, where $b>0$ is a constant.

Proof. Let $x$ be an eventually positive solution of Equation (1) that satisfies $\lim _{t \rightarrow \infty} x(t)=\infty$. Suppose that $\lim _{t \rightarrow \infty} z(t)<\infty$. Then, by Lemma $2, \lim _{t \rightarrow \infty} x(t)<\infty$, which causes a contradiction. Therefore, $\lim _{t \rightarrow \infty} z(t)=\infty$. In view of equality (17), letting $t \rightarrow \infty$, it follows that condition (5) is fulfilled. Define $R$ by (6). It follows from Theorem 1 that $x \in A(0)$ or $x \in A(b)$, where $b>0$ is a constant. The proof is complete.

Theorem 3. Equation (1) has an eventually positive solution which is in $A(b)$ iff

$$
\int_{t_{0}}^{\infty} f(t, K R(h(t))) \Delta t<\infty
$$

for some constant $K>0$, where $b>0$ is a constant.

Proof. Let $x \in A(b)$ be an eventually positive solution of Equation (1), where $b>0$ is a constant. By virtue of Lemma 2 and Theorem 1, we deduce that

$$
\lim _{t \rightarrow \infty} z(t)=\infty, \quad \lim _{t \rightarrow \infty} R_{n-1}(t, x(t))=\lim _{t \rightarrow \infty} \frac{z(t)}{R(t)}=\left(1+p_{0} \eta\right) b .
$$

There is a $t_{1} \in\left[t_{0}, \infty\right)_{\mathbb{T}}$ such that $x(t)>0, x(g(t))>0$, and $x(h(t)) \geq b R(h(t)) / 2$ for $t \in\left[t_{1}, \infty\right)_{\mathbb{T}}$. Integration of Equation (1) from $t_{1}$ to $s\left(s \in\left[\sigma\left(T_{1}\right), \infty\right)_{\mathbb{T}}\right)$ yields

$$
R_{n-1}(s, x(s))-R_{n-1}\left(t_{1}, x\left(t_{1}\right)\right)=-\int_{t_{1}}^{s} f(t, x(h(t))) \Delta t .
$$

Letting $s \rightarrow \infty$, it follows that

$$
\int_{t_{1}}^{\infty} f(t, x(h(t))) \Delta t<\infty
$$

Since $x(h(t)) \geq b R(h(t)) / 2$ for $t \in\left[t_{1}, \infty\right)_{\mathbb{T}}$, by $(C 4)$, we conclude that

$$
\int_{t_{1}}^{\infty} f\left(t, \frac{b}{2} R(h(t))\right) \Delta t \leq \int_{t_{1}}^{\infty} f(t, x(h(t))) \Delta t<\infty,
$$

which means that condition (20) holds.

Then, suppose that condition (20) holds for some constant $K>0$.

Case (i). $0 \leq p_{0}<1$. Choose a constant $p_{1}$ as in the proof of Theorem 2 . When $p_{0}>0$, there is a $T_{0} \in\left[t_{0}, \infty\right)_{\mathbb{T}}$ such that for $t \in\left[T_{0}, \infty\right)_{\mathbb{T}}$,

$$
\begin{gathered}
p(t)>0, \quad \frac{5 p_{1}-1}{4} \leq p(t) \leq p_{1}<1, \quad p(t) \frac{R(g(t))}{R(t)} \geq \frac{5 p_{1}-1}{4} \eta, \\
\int_{T_{0}}^{\infty} f(t, K R(h(t))) \Delta t \leq \frac{\left(1-p_{1} \eta\right) K}{8} .
\end{gathered}
$$


When $p_{0}=0$, take a constant $p_{1}$ satisfying $|p(t)| \leq p_{1} \leq 1 / 13$ for $t \in\left[T_{0}, \infty\right)_{\mathbb{T}}$. There also exists a $T_{1} \in\left(T_{0}, \infty\right)_{\mathbb{T}}$ such that $g(t) \geq T_{0}$ and $h(t) \geq T_{0}$ for $t \in\left[T_{1}, \infty\right)_{\mathbb{T}}$.

Let

$$
\Omega_{2}=\left\{x \in \mathrm{BC}_{1}\left[T_{0}, \infty\right)_{\mathbb{T}}: \frac{K}{2} R(t) \leq x(t) \leq K R(t)\right\} .
$$

Then, $\Omega_{2}$ is also a bounded, convex, and closed subset of $\mathrm{BC}_{1}\left[T_{0}, \infty\right)_{\mathbb{T}}$. Define now two operators $U_{2}, S_{2}: \Omega_{2} \rightarrow \mathrm{BC}_{1}\left[T_{0}, \infty\right)_{\mathbb{T}}$ by

$$
\begin{gathered}
\left(U_{2} x\right)(t)= \begin{cases}3 K p_{1} \eta R(t) / 4-p\left(T_{1}\right) x\left(g\left(T_{1}\right)\right) R(t) / R\left(T_{1}\right), & t \in\left[T_{0}, T_{1}\right)_{\mathbb{T}}, \\
3 K p_{1} \eta R(t) / 4-p(t) x(g(t)), & t \in\left[T_{1}, \infty\right)_{\mathbb{T}},\end{cases} \\
\left(S_{2} x\right)(t)= \begin{cases}3 K R(t) / 4, & t \in\left[T_{0}, T_{1}\right)_{\mathbb{T}}, \\
3 K R(t) / 4 & \\
+\int_{T_{1}}^{t} \int_{T_{1}}^{u_{n-1}} \int_{T_{1}}^{u_{n-2}} \cdots \int_{T_{1}}^{u_{2}} \int_{u_{1}}^{\infty} \frac{f\left(u_{0}, x\left(h\left(u_{0}\right)\right)\right)}{\prod_{i=1}^{n-1} r_{i}\left(u_{i}\right)} \Delta u_{0} \Delta u_{1} \cdots \Delta u_{n-1}, & t \in\left[T_{1}, \infty\right)_{\mathbb{T}} .\end{cases}
\end{gathered}
$$

The proof that $U_{2}$ and $S_{2}$ satisfy the conditions in Lemma 1 is also omitted. Similarly, there is an $x \in \Omega_{2}$ such that $\left(U_{2}+S_{2}\right) x=x$. For $t \in\left[T_{1}, \infty\right)_{\mathbb{T}}$,

$$
\begin{aligned}
x(t)= & \frac{3\left(1+p_{1} \eta\right) K}{4} R(t)-p(t) x(g(t)) \\
& +\int_{T_{1}}^{t} \int_{T_{1}}^{u_{n-1}} \int_{T_{1}}^{u_{n-2}} \cdots \int_{T_{1}}^{u_{2}} \int_{u_{1}}^{\infty} \frac{f\left(u_{0}, x\left(h\left(u_{0}\right)\right)\right)}{\prod_{i=1}^{n-1} r_{i}\left(u_{i}\right)} \Delta u_{0} \Delta u_{1} \cdots \Delta u_{n-1} .
\end{aligned}
$$

Letting $t \rightarrow \infty$, we conclude that

$$
\lim _{t \rightarrow \infty} \frac{z(t)}{R(t)}=\frac{3\left(1+p_{1} \eta\right) K}{4} \text { and } \quad \lim _{t \rightarrow \infty} \frac{x(t)}{R(t)}=\frac{3\left(1+p_{1} \eta\right) K}{4\left(1+p_{0} \eta\right)}>0
$$

which yields $\lim _{t \rightarrow \infty} x(t)=\infty$.

Case (ii). $-1<p_{0}<0$. Introduce $\mathrm{BC}_{1}\left[T_{0}, \infty\right)_{\mathbb{T}}$ and its subset $\Omega_{2}$ as in (21). Define $S_{2}$ by (22) and $U_{2}^{\prime}$ on $\Omega_{2}$ by

$$
\left(U_{2}^{\prime} x\right)(t)= \begin{cases}-3 K p_{1} \eta R(t) / 4-p\left(T_{1}\right) x\left(g\left(T_{1}\right)\right) R(t) / R\left(T_{1}\right), & t \in\left[T_{0}, T_{1}\right)_{\mathbb{T}}, \\ -3 K p_{1} \eta R(t) / 4-p(t) x(g(t)), & t \in\left[T_{1}, \infty\right)_{\mathbb{T}} .\end{cases}
$$

Similarly, $U_{2}^{\prime}$ and $S_{2}$ also satisfy the assumptions in Lemma 1 . There exists an $x \in \Omega_{2}$ such that $\left(U_{2}^{\prime}+S_{2}\right) x=x$. For $t \in\left[T_{1}, \infty\right)_{\mathbb{T}}$,

$$
\begin{aligned}
x(t)= & \frac{3\left(1-p_{1} \eta\right) K}{4} R(t)-p(t) x(g(t)) \\
& +\int_{T_{1}}^{t} \int_{T_{1}}^{u_{n-1}} \int_{T_{1}}^{u_{n-2}} \cdots \int_{T_{1}}^{u_{2}} \int_{u_{1}}^{\infty} \frac{f\left(u_{0}, x\left(h\left(u_{0}\right)\right)\right)}{\prod_{i=1}^{n-1} r_{i}\left(u_{i}\right)} \Delta u_{0} \Delta u_{1} \cdots \Delta u_{n-1} .
\end{aligned}
$$

Then, we deduce that

$$
\lim _{t \rightarrow \infty} \frac{z(t)}{R(t)}=\frac{3\left(1-p_{1} \eta\right) K}{4} \quad \text { and } \quad \lim _{t \rightarrow \infty} \frac{x(t)}{R(t)}=\frac{3\left(1-p_{1} \eta\right) K}{4\left(1+p_{0} \eta\right)}>0 .
$$

It follows that $\lim _{t \rightarrow \infty} x(t)=\infty$. This completes the proof.

Theorem 4. Assume that Equation (1) has an eventually positive solution which is in A(0). Then,

$$
\int_{t_{0}}^{\infty} f\left(t, \frac{3}{4}\right) \Delta t<\infty
$$


and

$$
\int_{t_{0}}^{\infty} \int_{t_{0}}^{u_{n-1}} \int_{t_{0}}^{u_{n-2}} \cdots \int_{t_{0}}^{u_{2}} \int_{u_{1}}^{\infty} \frac{f\left(u_{0}, R\left(h\left(u_{0}\right)\right)\right)}{\prod_{i=1}^{n-1} r_{i}\left(u_{i}\right)} \Delta u_{0} \Delta u_{1} \cdots \Delta u_{n-1}=\infty .
$$

Suppose that $|p(t) R(t)| \leq M$ for some constant $M>0$ and for $t \in\left[t_{0}, \infty\right)_{\mathbb{T}}$,

$$
\int_{t_{0}}^{\infty} f(t, R(h(t))) \Delta t<\infty
$$

and

$$
\int_{t_{0}}^{\infty} \int_{t_{0}}^{u_{n-1}} \int_{t_{0}}^{u_{n-2}} \cdots \int_{t_{0}}^{u_{2}} \int_{u_{1}}^{\infty} \frac{f\left(u_{0}, M+3 / 4\right)}{\prod_{i=1}^{n-1} r_{i}\left(u_{i}\right)} \Delta u_{0} \Delta u_{1} \cdots \Delta u_{n-1}=\infty .
$$

Then, Equation (1) has an eventually positive solution which is in $A(0)$.

Proof. Let $x \in A(0)$ be an eventually positive solution of Equation (1). Similarly, as in the proof of Theorem 3 , we arrive at

$$
\lim _{t \rightarrow \infty} z(t)=\infty, \quad \lim _{t \rightarrow \infty} R_{n-1}(t, x(t))=\lim _{t \rightarrow \infty} \frac{z(t)}{R(t)}=0 .
$$

There exist a $t_{1} \in\left[t_{0}, \infty\right)_{\mathbb{T}}$ and a $t_{2} \in\left(t_{1}, \infty\right)_{\mathbb{T}}$ such that $3 / 4 \leq x(t) \leq R(t)$ for $t \in\left[t_{1}, \infty\right)_{\mathbb{T}}$, and $g(t) \geq t_{1}, h(t) \geq t_{1}$ for $t \in\left[t_{2}, \infty\right)_{\mathbb{T}}$. Integration of Equation (1) from $t_{2}$ to $s\left(s \in\left[\sigma\left(t_{2}\right), \infty\right)_{\mathbb{T}}\right)$ yields

$$
R_{n-1}(s, x(s))-R_{n-1}\left(t_{2}, x\left(t_{2}\right)\right)=-\int_{t_{2}}^{s} f(t, x(h(t))) \Delta t .
$$

Letting $s \rightarrow \infty$, we obtain

$$
R_{n-1}\left(t_{2}, x\left(t_{2}\right)\right)=\int_{t_{2}}^{\infty} f(t, x(h(t))) \Delta t
$$

Since $x(t) \geq 3 / 4$ for $t \in\left[t_{2}, \infty\right)_{\mathbb{T}}$, by $(C 4)$, we get

$$
\int_{t_{2}}^{\infty} f\left(t, \frac{3}{4}\right) \Delta t<\infty
$$

which implies that inequality (23) holds. Then, replacing $t$ with $u_{0}$, and $t_{2}$ with $u_{1}$ in equality (27), it follows that

$$
R_{n-2}^{\Delta}\left(u_{1}, x\left(u_{1}\right)\right)=\frac{\int_{u_{1}}^{\infty} f\left(u_{0}, x\left(h\left(u_{0}\right)\right)\right) \Delta u_{0}}{r_{1}\left(u_{1}\right)} .
$$

Integrating equality (28) from $t_{2}$ to $u_{2}, u_{2} \in\left[\sigma\left(t_{2}\right), \infty\right)_{\mathbb{T}}$, we have

$$
R_{n-3}^{\Delta}\left(u_{2}, x\left(u_{2}\right)\right)=\frac{r_{2}\left(t_{2}\right) R_{n-3}^{\Delta}\left(t_{2}, x\left(t_{2}\right)\right)}{r_{2}\left(u_{2}\right)}+\frac{1}{r_{2}\left(u_{2}\right)} \int_{t_{2}}^{u_{2}} \int_{u_{1}}^{\infty} \frac{f\left(u_{0}, x\left(h\left(u_{0}\right)\right)\right)}{r_{1}\left(u_{1}\right)} \Delta u_{0} \Delta u_{1} .
$$

Analogously, for $n \geq 3$, we conclude that

$$
\begin{aligned}
z(t)-z\left(t_{2}\right)= & R_{1}\left(t_{2}, x\left(t_{2}\right)\right) \int_{t_{2}}^{t} \frac{1}{r_{n-1}\left(u_{n-1}\right)} \Delta u_{n-1} \\
& +\sum_{k=2}^{n-1} R_{k}\left(t_{2}, x\left(t_{2}\right)\right) \int_{t_{2}}^{t} \int_{t_{2}}^{u_{n-1}} \int_{t_{2}}^{u_{n-2}} \cdots \int_{t_{2}}^{u_{n-k+1}} \frac{1}{\prod_{i=n-k}^{n-1} r_{i}\left(u_{i}\right)} \Delta u_{n-k} \Delta u_{n-k+1} \cdots \Delta u_{n-1} \\
& +\int_{t_{2}}^{t} \int_{t_{2}}^{u_{n-1}} \int_{t_{2}}^{u_{n-2}} \cdots \int_{t_{2}}^{u_{2}} \int_{u_{1}}^{\infty} \frac{f\left(u_{0}, x\left(h\left(u_{0}\right)\right)\right)}{\prod_{i=1}^{n-1} r_{i}\left(u_{i}\right)} \Delta u_{0} \Delta u_{1} \cdots \Delta u_{n-1} .
\end{aligned}
$$


Letting $t \rightarrow \infty$, similarly as the proof in Theorem 2 , we deduce that

$$
\int_{t_{2}}^{\infty} \int_{t_{2}}^{u_{n-1}} \int_{t_{2}}^{u_{n-2}} \cdots \int_{t_{2}}^{u_{2}} \int_{u_{1}}^{\infty} \frac{f\left(u_{0}, x\left(h\left(u_{0}\right)\right)\right)}{\prod_{i=1}^{n-1} r_{i}\left(u_{i}\right)} \Delta u_{0} \Delta u_{1} \cdots \Delta u_{n-1}=\infty .
$$

Since $x(t) \leq R(t)$ for $t \in\left[t_{2}, \infty\right)_{\mathbb{T}}$, by virtue of $(C 4)$,

$$
\int_{t_{2}}^{\infty} \int_{t_{2}}^{u_{n-1}} \int_{t_{2}}^{u_{n-2}} \cdots \int_{t_{2}}^{u_{2}} \int_{u_{1}}^{\infty} \frac{f\left(u_{0}, R\left(h\left(u_{0}\right)\right)\right)}{\prod_{i=1}^{n-1} r_{i}\left(u_{i}\right)} \Delta u_{0} \Delta u_{1} \cdots \Delta u_{n-1}=\infty,
$$

which means that equality (24) holds.

Assume that $|p(t) R(t)| \leq M$ for some constant $M>0$ and for $t \in\left[t_{0}, \infty\right)_{\mathbb{T}}$, conditions (25) and (26) hold. Then, $\lim _{t \rightarrow \infty} p(t)=p_{0}=0$. Choose a $T_{0} \in\left[t_{0}, \infty\right)_{\mathbb{T}}$ and $0<p_{1}<1$ such that for $t \in\left[T_{0}, \infty\right)_{\mathbb{T}}$,

$$
|p(t)| \leq p_{1}<1, \quad 2 M+\frac{3}{2} \leq \frac{1}{4} R(t), \quad \int_{T_{0}}^{\infty} f(t, R(h(t))) \Delta t \leq \frac{1-p_{1}}{8} .
$$

There exists a $T_{1} \in\left(T_{0}, \infty\right)_{\mathbb{T}}$ such that $g(t) \geq T_{0}$ and $h(t) \geq T_{0}$ hold for $t \in\left[T_{1}, \infty\right)_{\mathbb{T}}$.

Let

$$
\Omega_{3}=\left\{x \in \mathrm{BC}_{1}\left[T_{0}, \infty\right)_{\mathbb{T}}: M+\frac{3}{4} \leq x(t) \leq R(t)\right\} .
$$

Then, $\Omega_{3}$ is a bounded, convex, and closed subset of $\mathrm{BC}_{1}\left[T_{0}, \infty\right)_{\mathbb{T}}$. Define now two operators $U_{3}, S_{3}: \Omega_{3} \rightarrow \mathrm{BC}_{1}\left[T_{0}, \infty\right)_{\mathbb{T}}$ by

$$
\begin{gathered}
\left(U_{3} x\right)(t)= \begin{cases}M+3 / 4-p\left(T_{1}\right) x\left(g\left(T_{1}\right)\right) R(t) / R\left(T_{1}\right), & t \in\left[T_{0}, T_{1}\right)_{\mathbb{T}}, \\
M+3 / 4-p(t) x(g(t)), & t \in\left[T_{1}, \infty\right)_{\mathbb{T}},\end{cases} \\
\left(S_{3} x\right)(t)=\left\{\begin{array}{l}
M+3 / 4, \\
M+3 / 4 \\
\left.+\int_{T_{1}}^{t} \int_{T_{1}}^{u_{n-1}} \int_{T_{1}}^{u_{n-2}} \cdots \int_{T_{1}}^{u_{2}} \int_{u_{1}}^{\infty} \frac{f\left(u_{0}, x\left(h\left(u_{0}\right)\right)\right)}{\prod_{i=1}^{n-1} r_{i}\left(u_{i}\right)} \Delta u_{1}\right)_{\mathbb{T}},
\end{array}\right.
\end{gathered}
$$

The proof that $U_{3}$ and $S_{3}$ satisfy the assumptions in Lemma 1 is also omitted. Then, there is an $x \in \Omega_{3}$ such that $\left(U_{3}+S_{3}\right) x=x$. For $t \in\left[T_{1}, \infty\right)_{\mathbb{T}}$,

$$
x(t)=2 M+\frac{3}{2}-p(t) x(g(t))+\int_{T_{1}}^{t} \int_{T_{1}}^{u_{n-1}} \int_{T_{1}}^{u_{n-2}} \cdots \int_{T_{1}}^{u_{2}} \int_{u_{1}}^{\infty} \frac{f\left(u_{0}, x\left(h\left(u_{0}\right)\right)\right)}{\prod_{i=1}^{n-1} r_{i}\left(u_{i}\right)} \Delta u_{0} \Delta u_{1} \cdots \Delta u_{n-1} .
$$

In view of condition (26), we get

$$
\lim _{t \rightarrow \infty} z(t)=\infty \text { and } \lim _{t \rightarrow \infty} \frac{z(t)}{R(t)}=0 .
$$

Since $|p(t) x(g(t))| \leq|p(t) R(t)| \leq M$, by virtue of Lemma 2, we conclude that

$$
\lim _{t \rightarrow \infty} x(t)=\infty \quad \text { and } \quad \lim _{t \rightarrow \infty} \frac{x(t)}{R(t)}=0
$$

The proof is complete.

Remark 2. It is not easy to establish the sufficient and necessary conditions which guarantee that Equation (1) has an eventually positive solution $x$ satisfying $\lim _{t \rightarrow \infty} x(t)=0$. We refer the reader to [20] (Theorems 3.2 and 3.3) for sufficient conditions to ensure it. 
Remark 3. When $n=3$, it is obvious that Theorems 2-4, Corollary 1, and Lemma 3 cover the results in [22]. Furthermore, even when $n=2$, the conclusions above are also consistent with those in [12,13].

\section{Examples}

The following two examples are presented to illustrate theoretical results obtained in this article.

Example 1. Let $\mathbb{T}=\bigcup_{n=1}^{\infty}[(4 n-3) c, 4 n c]$, where $c>0$. For $t \in[5 c, \infty)_{\mathbb{T}}$, consider

$$
R_{n}(t, x(t))+t^{\gamma} x(t)=0,
$$

where $n \geq 3, \gamma \in \mathbb{R}$, and

$$
R_{k}(t, x(t))=\left\{\begin{array}{lc}
x(t)-(t-c) /(2 t) x(t-4 c), & k=0, \\
t^{n-k} R_{k-1}^{\Delta}(t, x(t)), & 1 \leq k \leq n-1, \\
R_{n-1}^{\Delta}(t, x(t)), & k=n .
\end{array}\right.
$$

We can see that $r_{i}(t)=t^{i}, i=1,2, \ldots, n-1, p(t)=-(t-c) /(2 t), g(t)=t-4 c, h(t)=t$, $f(t, x)=t^{\gamma} x, t_{0}=5 c$, and $p_{0}=-1 / 2$. Obviously, conditions (C1)-(C4) and (14) are satisfied. Taking $K=1$, we conclude that

$$
\begin{aligned}
& \int_{t_{0}}^{\infty} \int_{t_{0}}^{u_{n-1}} \int_{t_{0}}^{u_{n-2}} \cdots \int_{t_{0}}^{u_{1}} \frac{f\left(u_{0}, 1\right)}{\prod_{i=1}^{n-1} r_{i}\left(u_{i}\right)} \Delta u_{0} \Delta u_{1} \cdots \Delta u_{n-1} \\
= & \int_{5 c}^{\infty} \int_{5 c}^{u_{n-1}} \int_{5 c}^{u_{n-2}} \cdots \int_{5 c}^{u_{1}} \frac{u_{0}^{\gamma}}{\prod_{i=1}^{n-1} u_{i}^{i}} \Delta u_{0} \Delta u_{1} \cdots \Delta u_{n-1} \\
= & \int_{5 c}^{\infty} O\left(u_{n-1}^{\gamma-(n-1)(n-2) / 2}\right) \Delta u_{n-1} .
\end{aligned}
$$

When $\gamma<n(n-3) / 2$, which means that $\gamma-(n-1)(n-2) / 2<-1$, condition (15) holds. By virtue of Theorem 2 (or Corollary 1), we deduce that Equation (29) has an eventually positive solution $x$ that satisfies $\lim _{t \rightarrow \infty} x(t)=b$, where $b>0$ is a constant. Moreover, Equation (29) has no eventually positive solutions $x$ satisfying $\lim _{t \rightarrow \infty} x(t)=b>0$ provided that $\gamma \geq n(n-3) / 2$.

Example 2. Let $\mathbb{T}=\bigcup_{n=0}^{\infty}\left[2 \cdot 3^{n}, 3^{n+1}\right]$. For $t \in[6, \infty)_{\mathbb{T}}$, consider

$$
R_{n}(t, x(t))+t^{\gamma} x^{3}(3 t)=0,
$$

where $n \geq 3, \gamma \in \mathbb{R}$, and

$$
R_{k}(t, x(t))=\left\{\begin{array}{lc}
x(t)+1 / t \cdot x(t / 3), & k=0 \\
t^{2} R_{k-1}^{\Delta}(t, x(t)), & 1 \leq k \leq n-2 \\
1 / t^{n-2} \cdot R_{n-2}^{\Delta}(t, x(t)), & k=n-1 \\
R_{n-1}^{\Delta}(t, x(t)), & k=n
\end{array}\right.
$$

We get $r_{1}(t)=1 / t^{n-2}, r_{i}(t)=t^{2}, i=2,3, \ldots, n-1, p(t)=1 / t, g(t)=t / 3, h(t)=3 t, f(t, x)=$ $t^{\gamma} x^{3}, t_{0}=6$, and $p_{0}=0$. It is not difficult to see that the assumptions $(C 1)-(C 4)$ are fulfilled. From $(C 5)$, we have

$$
\begin{aligned}
R(t) & =1+\int_{t_{0}}^{t} \int_{t_{0}}^{u_{n-1}} \int_{t_{0}}^{u_{n-2}} \cdots \int_{t_{0}}^{u_{2}} \frac{1}{\prod_{i=1}^{n-1} r_{i}\left(u_{i}\right)} \Delta u_{1} \Delta u_{2} \cdots \Delta u_{n-1} \\
& =1+\int_{6}^{t} \int_{6}^{u_{n-1}} \int_{6}^{u_{n-2}} \cdots \int_{6}^{u_{2}} \frac{u_{1}^{n-2}}{\prod_{i=2}^{n-1} u_{i}^{2}} \Delta u_{1} \Delta u_{2} \cdots \Delta u_{n-1}=O(t),
\end{aligned}
$$


which implies that

$$
\eta=\lim _{t \rightarrow \infty} \frac{R(g(t))}{R(t)}=\lim _{t \rightarrow \infty} \frac{O(t / 3)}{O(t)}=\frac{1}{3} \in(0,1] .
$$

Hence, (C5) holds, and we arrive at

$$
\int_{t_{0}}^{\infty} f(t, K R(h(t))) \Delta t=\int_{6}^{\infty} t^{\gamma}(O(t))^{3} \Delta t=\int_{6}^{\infty} O\left(t^{\gamma+3}\right) \Delta t
$$

Due to Theorem 3, condition (20) holds when $\gamma<-4$, and we conclude that Equation (30) has an eventually positive solution $x \in A(b)$ for some constant $b>0$. However, Equation (30) has no eventually positive solutions $x \in A(b)$ provided that $\gamma \geq-4$.

On the other hand, when $\gamma<-5$, we obtain

$$
\begin{aligned}
& \int_{t_{0}}^{\infty} \int_{t_{0}}^{u_{n-1}} \int_{t_{0}}^{u_{n-2}} \cdots \int_{t_{0}}^{u_{2}} \int_{u_{1}}^{\infty} \frac{f\left(u_{0}, R\left(h\left(u_{0}\right)\right)\right)}{\prod_{i=1}^{n-1} r_{i}\left(u_{i}\right)} \Delta u_{0} \Delta u_{1} \cdots \Delta u_{n-1} \\
= & \int_{6}^{\infty} \int_{6}^{u_{n-1}} \int_{6}^{u_{n-2}} \cdots \int_{6}^{u_{2}} \int_{u_{1}}^{\infty} \frac{O\left(u_{0}^{\gamma+3}\right) \cdot u_{1}^{n-2}}{\prod_{i=2}^{n-1} u_{i}^{2}} \Delta u_{0} \Delta u_{1} \cdots \Delta u_{n-1} \\
= & \int_{6}^{\infty} u_{n-1}^{\gamma+4} \Delta u_{n-1}<\infty .
\end{aligned}
$$

That is, inequality (23) does not hold. It follows from Theorem 4 that Equation (30) has no eventually positive solutions $x \in A(0)$.

Author Contributions: All four authors contributed equally to this work. They all read and approved the final version of the manuscript.

Funding: The research was supported by the National Natural Science Foundation of P. R. China (Grant Nos. 11671406 and 61503171), the China Postdoctoral Science Foundation (Grant No. 2015M582091), the Natural Science Program for Young Creative Talents of Innovation Enhancing College Project of Department of Education of Guangdong Province (Grant Nos. 2017GKQNCX111 and 2018-KJZX039), the Natural Science Foundation of Shandong Province (Grant No. ZR2016JL021), and the Key Research and Development Program of Shandong Province (Grant No. 2017CXGC0701).

Acknowledgments: The authors express their sincere gratitude to the editors and three anonymous referees for the careful reading of the original manuscript and valuable suggestions that helped to improve the article.

Conflicts of Interest: The authors declare that they have no conflicts of interest.

\section{References}

1. Hilger, S. Ein Maßkettenkalkül mit Anwendung auf Zentrumsmannigfaltigkeiten. Ph.D. Thesis, Universität Würzburg, Würzburg, Germany, 1988.

2. Hilger, S. Analysis on measure chains-A unified approach to continuous and discrete calculus. Results Math. 2000, 18, 18-56. [CrossRef]

3. Agarwal, R.P.; Bohner, M. Basic calculus on time scales and some of its applications. Results Math. 1999, 35, 3-22. [CrossRef]

4. Agarwal, R.P.; Bohner, M.; O’Regan, D.; Peterson, A. Dynamic equations on time scales: A survey. J. Comput. Appl. Math. 2002, 141, 1-26. [CrossRef]

5. Bohner, M.; Peterson, A. Dynamic Equations on Time Scales: An Introduction with Applications; Birkhäuser: Boston, MA, USA, 2001.

6. Bohner, M.; Peterson, A. Advances in Dynamic Equations on Time Scales; Birkhäuser: Boston, MA, USA, 2003.

7. Agarwal, R.P.; Bohner, M.; Li, T.X.; Zhang, C.H. Hille and Nehari type criteria for third-order delay dynamic equations. J. Differ. Equat. Appl. 2013, 19, 1563-1579. [CrossRef]

8. Agarwal, R.P.; Bohner, M.; Li, T.X.; Zhang, C.H. A Philos-type theorem for third-order nonlinear retarded dynamic equations. Appl. Math. Comput. 2014, 249, 527-531. [CrossRef] 
9. Agarwal, R.P.; Bohner, M.; Tang, S.H.; Li, T.X.; Zhang, C.H. Oscillation and asymptotic behavior of third-order nonlinear retarded dynamic equations. Appl. Math. Comput. 2012, 219, 3600-3609. [CrossRef]

10. Bohner, M.; Li, T.X. Oscillation of second-order $p$-Laplace dynamic equations with a nonpositive neutral coefficient. Appl. Math. Lett. 2014, 37, 72-76. [CrossRef]

11. Deng, X.H.; Wang, Q.R. Nonoscillatory solutions to forced higher-order nonlinear neutral dynamic equations on time scales. Rocky Mt. J. Math. 2015, 45, 475-507. [CrossRef]

12. Deng, X.H.; Wang, Q.R. Nonoscillatory solutions to second-order neutral functional dynamic equations on time scales. Commun. Appl. Anal. 2014, 18, 261-280.

13. Gao, J.; Wang, Q.R. Existence of nonoscillatory solutions to second-order nonlinear neutral dynamic equations on time scales. Rocky Mt. J. Math. 2013, 43, 1521-1535. [CrossRef]

14. Karpuz, B. Sufficient conditions for the oscillation and asymptotic behaviour of higher-order dynamic equations of neutral type. Appl. Math. Comput. 2013, 221, 453-462.

15. Karpuz, B.; Öcalan, Ö. Necessary and sufficient conditions on asymptotic behaviour of solutions of forced neutral delay dynamic equations. Nonlinear Anal. 2009, 71, 3063-3071. [CrossRef]

16. Li, T.X.; Han, Z.L.; Sun, S.R.; Yang, D.W. Existence of nonoscillatory solutions to second-order neutral delay dynamic equations on time scales. Adv. Differ. Equat. 2009, 2009, 562329. [CrossRef]

17. Li, T.X.; Saker, S.H. A note on oscillation criteria for second-order neutral dynamic equations on isolated time scales. Commun. Nonlinear Sci. Numer. Simul. 2014, 19, 4185-4188. [CrossRef]

18. Mathsen, R.M.; Wang, Q.R.; Wu, H.W. Oscillation for neutral dynamic functional equations on time scales. J. Differ. Equat. Appl. 2004, 10, 651-659. [CrossRef]

19. Qiu, Y.C. Nonoscillatory solutions to third-order neutral dynamic equations on time scales. Adv. Differ. Equat. 2014, 2014, 309. [CrossRef]

20. Qiu, Y.C.; Wang, Q.R. Existence of nonoscillatory solutions to higher-order nonlinear neutral dynamic equations on time scales. Bull. Malays. Math. Sci. Soc. 2018, 41, 1935-1952. [CrossRef]

21. Qiu, Y.C.; Wang, H.X.; Jiang, C.M.; Li, T.X. Existence of nonoscillatory solutions to third-order neutral functional dynamic equations on time scales. J. Nonlinear Sci. Appl. 2018, 11, 274-287. [CrossRef]

22. Qiu, Y.C.; Zada, A.; Tang, S.H.; Li, T.X. Existence of nonoscillatory solutions to nonlinear third-order neutral dynamic equations on time scales. J. Nonlinear Sci. Appl. 2017, 10, 4352-4363. [CrossRef]

23. Zhang, C.H.; Agarwal, R.P.; Bohner, M.; Li, T.X. Oscillation of second-order nonlinear neutral dynamic equations with noncanonical operators. Bull. Malays. Math. Sci. Soc. 2015, 38, 761-778. [CrossRef]

24. Zhu, Z.Q.; Wang, Q.R. Existence of nonoscillatory solutions to neutral dynamic equations on time scales. J. Math. Anal. Appl. 2007, 335, 751-762. [CrossRef] 